\title{
RESPUESTA DE Bradyrhizobium japonicum A LA ADICIÓN DE ALGINATO EN PRESENCIA DE FUNGICIDAS PELETIZADOS EN SEMILLAS DE SOYA
}

\section{RESPONSE OF Bradyrhizobium japonicum TO ALGINATE IN PRESENCE OF PELLETED FUNGICIDES ON SOYBEAN SEEDS}

\author{
Felipe Andrés Romero-Perdomo ${ }^{1}$, Mauricio Camelo ${ }^{2}$, Ruth Bonilla ${ }^{3}$
}

\begin{abstract}
${ }^{1}$ BSc., Investigador profesional. Corporación Colombiana de Investigación Agropecuaria - CORPOICA-, e-mail: fromerop@ corpoica.org.co; ${ }^{2}$ BSc., Investigador profesional. Corporación Colombiana de Investigación Agropecuaria -CORPOICA-, e-mail: mcamelo@corpoica.org.co; ${ }^{3}$ Ph.D., Investigadora. Corporación Colombiana de Investigación Agropecuaria CORPOICA-, Mosquera/Colombia. Grupo de Investigación de Microbiología de Suelos, Centro de Biotecnología y Bioindustria (CBB), e-mail: rbonilla@corpoica.org.co [Autor para correspondencia]
\end{abstract}

Rev. U.D.C.A Act. \& Div. Cient. 18(2): 359-364, Julio-Diciembre, 2015

\section{RESUMEN}

El objetivo de la presente investigación fue determinar la respuesta de un inoculante con base en cepas de Bradyrhizobium japonicum a la adición de alginato, en presencia de los fungicidas Carbendazim ${ }^{\circledR}$ y Fludioxonil ${ }^{\circledR}$, peletizados en semillas de soya. El experimento consistió de ocho tratamientos completamente al azar, con un arreglo factorial $3 \times 2$, con tres repeticiones por triplicado. La estimación de sobrevivencia bacteriana, como variable de respuesta, fue realizada en tres tiempos de secado: 0, 24 y 48 horas. Los datos fueron expresados como [ $\log _{10} \mathrm{UFC} / \mathrm{mL}$ ]. Los resultados mostraron que los dos fungicidas reducen significativamente $(\mathrm{p}<0.05)$ la viabilidad de $B$. japonicum. El fungicida Fludioxonil ${ }^{\circledR}$ presentó mayor efecto inhibitorio que Carbendazim ${ }^{\circledR}$, con más del $25 \%$ de reducción, a las 48 horas. Además, se demostró que el alginato promueve la adherencia sobre las semillas de soya y la protección de las bacterias, disminuyendo la acción inhibitoria $(p<0.05)$, después de las 24 horas. Estos resultados sugieren que la aplicación de polímeros en inoculantes bacterianos representa una alternativa promisoria, para disminuir los efectos nocivos de fungicidas, sobre su viabilidad celular.

Palabras clave: Inoculantes, biopolímeros, Benzimidazol, Fenilpirrol.

\section{SUMMARY}

The aim of this research was to determine the response of an inoculant based on strains of Bradyrhizobium japonicum with the addition of alginate in presence of Carbendazim ${ }^{\circledR}$ and Fludioxonil ${ }^{\circledR}$ pelleted fungicides in soybean seeds. The experiment consisted of eight treatments completely randomized with a $3 \times 2$ factorial arrangement with three replications by triplicate. Estimation of bacterial survival, as response variable, was performed at three times of drying: 0, 24 and 48 hours. Data were expressed as Log10 (CFU/ $\mathrm{mL})$. Results showed that the two fungicides significantly reduce $(\mathrm{p}<0.05)$ the viability of $B$. japonicum. Fludioxonil ${ }^{\circledR}$ presented a stronger inhibitory effect than Carbendazim ${ }^{\circledR}$ with more than $25 \%$ of reduction at 48 hours. Moreover, it was shown that alginate promotes the adherence over soybean seeds and bacteria protection, diminishing the fungicide inhibitory action $(p<0.05)$ after 24 hours. These results suggest that application of polymers in bacteria inoculants are a promising alternative to reduce negative effects of fungicides over cellular viability.

Key words: Inoculants, biopolymer, Benzimidazole, Phenylpyrrole.

\section{INTRODUCCIÓN}

La soya es una leguminosa oleaginosa, que se ha constituido en materia prima esencial en la fabricación de concentrados balanceados, para la alimentación animal y es una buena alternativa para la rotación de cultivos, en el trópico colombiano. El área sembrada de esta especie, a nivel nacional, es aproximadamente de 27.480 hectáreas, con una producción de 53.100 toneladas y un rendimiento de 1,9t/ha (Valencia \& Ligarreto, 2010). A pesar de su impacto, su sistema de producción presenta una dependencia a la aplicación de diversos productos químicos, como el Carbendazim ${ }^{\circledR}$ (Me- 
til-2- bencimidazoil-carbamato) y el Fludioxonil ${ }^{\circledR}$ (4-(2,2-difluoro-1,3-benzodioxol-4-il)pirrol-3-carbonitrilo) (Veloukas et al. 2014). Estos son fungicidas comúnmente usados para el control y la prevención de enfermedades causadas por géneros, como Colletotrichum, Penicillium y Cladosporium, que atacan a las semillas y a las plántulas (Jianya et al. 2014). Diversas investigaciones han afirmado que algunos fungicidas pueden afectar negativamente la proliferación de microorganismos benéficos del suelo, como las bacterias promotoras de crecimiento vegetal (BPCV) (Ahemad \& Khan, 2012a; Miñambres et al. 2010; Lo, 2010).

Las BPCV son microorganismos que pueden crecer en o alrededor de los tejidos de las plantas, estimulando su crecimiento por diversos mecanismos, como la fijación biológica de nitrógeno, la solubilización y la mineralización de fosfato, la producción de indoles, sidérofos, entre otros (Pii et al. 2015); por estas razones, dichos microorganismos benéficos han sido considerados como agentes de fertilización biológica. Dentro de los géneros bacterianos más estudiados para promover el crecimiento de la soya, se encuentra Bradyrhizobium japonicum (Nguyen et al. 2012); su potencialidad, como biofertilizante, ha sido demostrada con reducciones mayores al $70 \%$ de fertilización nitrógenada química, representando una alternativa promisoria, para el desarrollo sostenible e integrado de este cultivo (Cassán et al. 2009).

Dentro de las formulaciones de inoculantes biológicos, se están estudiando el uso de biomateriales, para brindar mayor protección, estabilidad y conservación genética a las cepas (Cortés-Patiño \& Bonilla, 2015). Los biomateriales más empleados son los polímeros, como polivinilpirrolidona, goma acacia, carragenina, alginato y polietilenglicol (Rivera et al. 2014; Rojas-Tapias et al. 2013; Rojas-Tapias et al. 2015), que provienen del metabolismo de diversos microorganismos, paredes celulares de las algas marinas y resina de árboles (John et al. 2011). Existen varios requisitos para que estos polímeros sean componentes de inoculantes, como: ser no tóxicos y libre de conservantes perjudiciales; proteger a los microorganismos inoculados de competidores de suelo y de factores ambientales y ser lentamente degradables en el suelo y contener suficiente agua para la supervivencia y movimiento de los microorganismos (Bashan et al. 2013; Covarrubias et al. 2012). Es por esto, que algunos autores han propuesto el uso de polímeros como solución para mitigar el efecto perjudicial producido por fungicidas hacia las BPCV (Romero-Perdomo et al. 2015; Dayamani \& Brahmaprakash, 2014; Giongo et al. 2013).

El objetivo del presente estudio fue evidenciar la respuesta de la viabilidad celular de un inoculante, con base en cepas de $B$. japonicum, ante el uso del polímero alginato, en presencia de los fungicidas Carbendazim ${ }^{\circledR}$ y Fludioxonil ${ }^{\circledR}$, peletizados en semillas de soya, a diferentes tiempos de secado.

\section{MATERIALES Y MÉTODOS}

Microorganismos y condiciones de cultivo: En este estudio, se emplearon las cepas J01 y J96, identificadas molecularmente, con base en sus secuencias 16S rDNA, como B. japonicum; las cepas fueron reactivadas en YM (Vincent, 1970). Posteriormente, se realizó un pre inóculo en medio YM, a condiciones estándar: $30 \pm 2^{\circ} \mathrm{C}$ y 150 rpm.

La estandarización del inóculo de cada cepa, se realizó en medio YM líquido, empleando $1 \%$ (v/v) de pre inóculo. Las condiciones de incubación estándar fueron: $28 \pm 2^{\circ} \mathrm{C}$, 96h y 150rpm. La cuantificación de [ $\log _{10} \mathrm{UFC} / \mathrm{mL}$ ], se efectuó en medio YM más rojo congo $(10 \mathrm{~mL} / \mathrm{L})$, por la técnica de microgota y diluciones seriadas (Doyle et al. 2001). Las placas fueron incubadas en condiciones aeróbicas, a $30 \pm$ $2^{\circ} \mathrm{C}$, durante $96 \mathrm{~h}$; por último, se mezcló el inóculo de cada cepa, a una relación 1:1.

Respuesta de $B$. japonicum ante la adición de alginato en presencia de Carbendazim y Fludioxonil: Se aplicó un diseño completamente al azar con un arreglo factorial de $3 \times 2$, compuesto por ocho tratamientos por triplicado (Tabla 1). Los agroquímicos evaluados fueron Carbendazim ${ }^{\circledR}$ (Arysta LifeScience, France) y Fludioxonil ${ }^{\circledR} \quad$ (Syngenta AG, Switzerland) y el polímero empleado fue alginato (Biopolímeros del FMC- Ewing, EE.UU.). La variable de respuesta fue viabilidad celular [ $\log _{10} \mathrm{UFC} / \mathrm{mL}$ ] cuantificada, mediante el método de recuento en placa, con diluciones seriadas a las 0,24 y 48 horas de secado, a $30 \pm 2^{\circ} \mathrm{C}$.

Tabla 1. Efecto del alginato sobre la interacción fungicida - $B$. japonicum. Tratamientos evaluados.

\begin{tabular}{|c|l|}
\hline Identificación & \multicolumn{1}{|c|}{ Tratamientos } \\
\hline T1 & Testigo químico con Carbendazim ${ }^{\circledR}$ \\
\hline T2 & Testigo químico con Fludioxonil ${ }^{\circledR}$ \\
\hline T3 & Sin fungicida + inoculación sin polímero \\
\hline T4 & Sin fungicida + inoculación con polímero \\
\hline T5 & Carbendazim ${ }^{\circledR}+$ inoculación sin polímero \\
\hline T6 & Carbendazim ${ }^{\circledR}+$ inoculación con polímero \\
\hline T7 & Fludioxonil ${ }^{\circledR}+$ inoculación sin polímero \\
\hline T8 & Fludioxonil ${ }^{\circledR}+$ inoculación con polímero \\
\hline
\end{tabular}

Inmovilización en polímero: Se usó alginato al 3,0\% (m/v) mezclado con agua destilada; la relación del alginato respecto el inóculo de las cepas fue 1:10, respectivamente. 
Proceso de peletización: Se desinfectaron las semillas de soya sumergiéndolas en peróxido de hidrógeno 30\% y etanol $70 \%$ por 10 min cada uno, seguido por enjuagues consecutivos, con agua destilada estéril (Rojas-Tapias et al. 2012). Posteriormente, se mezclaron las semillas con los fungicidas Carbendazim ${ }^{\circledR}(0,5 \mathrm{~L}$ de fungicida/kg semilla/L agua) y Fludioxonil ${ }^{\circledR}$ (1,5L de fungicida/kg semilla/L agua); después, se realizó el proceso de secado, a temperatura ambiente, durante 60 minutos y, por último, se procedió a la adhesión in vitro de las semillas con el inóculo, con una dosis de $15 \mathrm{~kg}$ de semilla/L.

Análisis estadístico: Los datos obtenidos fueron analizados a través de ANOVA univariante de multivías y test HSDTukey, mediante el paquete estadístico SPSS 17; todos los experimentos, se llevaron a cabo con un 95\% de nivel de confianza. Las figuras fueron realizadas con Sigmaplot 11.0.

\section{RESULTADOS Y DISCUSIÓN}

Los resultados revelaron que los dos fungicidas afectaron significativamente $(\mathrm{p}<0.05)$ la viabilidad celular de $B$. japonicum. Una disminución progresiva de la viabilidad, a través del tiempo, se observó en el T5 (Carbendazim ${ }^{\circledR}$ más $B$. japonicum sin alginato) y T7 (Fludioxonil ${ }^{\circledR}$ más $B$. japonicum sin alginato). Resultados diferentes fueron encontrados en T3 (sin fungicida y sin polímero), donde se evidenció que la población bacteriana, se mantuvo constante; los recuentos de viabilidad celular obtenidos en T5 y T7 siempre fueron inferiores respecto a T3. Específicamente, a las 48 horas, se cuantificaron pérdidas de más de dos unidades logarítmicas de biomasa en T5 y T7 respecto al T3 (Figura 1).

Entre los dos fungicidas, el Fludioxonil ${ }^{\circledR}$ afectó más la viabilidad de $B$.japonicum, generando $26 \%$ de pérdida celular a las 48 horas de secado. Imfelda \& Vuilleumierb (2012) afirman que los fungicidas pueden generar diferentes efectos en BPCV; por ejemplo, Deshwal et al. (2013) y Ahemad \& Khan (2012b) reportaron una versatilidad metabólica del género Bradyrhizobium sp., para utilizar sustancias aromáticas cloradas, como fuente de energía. En contraste, otras investigaciones indican que el contacto con compuestos, como el benomil, carbendazim y captan, reducen entre un $14-45 \%$ las poblaciones de $B$. japonicum (Rubens et al. 2009; Mishra et al. 2013). De lo poco que se sabe respecto al efecto del Fludioxonil ${ }^{\circledR}$ sobre el metabolismo de BPCV, se ha reportado la inhibición de la síntesis de pirrolnitrina en Burkholderia sp. O33 y Pseudomonas fluorescens Pf-5 (Keum et al. 2010). Posiblemente, su sustancia activa, el Fenilpirrol, afecte mecanismos de intercambios celulares dependientes de la membrana, bloqueando el crecimiento $B$. japonicum, como ocurre a nivel fúngico (Zhang et al. 2002).
Por otro lado, la presencia de alginato frente a la interacción semilla-microorganismo-fungicida, también presentó efecto significativo $(p<0.05)$ sobre la biomasa de la cepas. Los resultados encontrados entre T3 y T4 indicaron que el alginato favorece la adherencia entre la superficie de la semilla y el material celular. A la hora 0 de secado, se evidenciaron diferencias significativas $(p<0.05)$ entre estos tratamientos que no contenían fungicida (Figura 1A). Las 0,8 [ $\log _{10} \mathrm{UFC} / \mathrm{mL}$ ] cuantificadas demás en el T4 están atribuidas a la estructura química del alginato; el alginato es un copolímero compuesto por los ácidos D-manúronico (M) y L-gulurónico (G). Los grupos carboxilos terminales de $M$ y G generan un carácter aniónico a la estructura que le proporciona propiedad mucoadherente (Lee \& Mooney, 2012).

Otro efecto ejercido por el alginato, se evidenció entre los tratamientos 5 y 6 y 7 y 8 . Las [ $\log _{10}(\mathrm{UFC} / \mathrm{mL}$ ] cuantificadas reflejaron una protección por parte de este polímero sobre $B$. japonicum; el efecto protector del alginato hacia el Carbendazim ${ }^{\circledR}$ fue observado entre $\mathrm{T} 5$ y T6. Existieron diferencias significativas $(p<0.05)$ a las 48 horas de secado entre estos dos tratamientos (Figuras 1B y 1C), donde la biomasa celular fue mayor en $31 \%$ en presencia de alginato; asimismo, los resultados con Fludioxonil ${ }^{\circledR}$ entre T7 y T8 mostraron un comportamiento similar. La protección del alginato frente a la acción perjudicial de este fungicida sobre la biomasa fue del 25\%, a las 48 horas. Según Gombotz \& Wee (2012), el efecto protector del alginato depende de la viscosidad y de la fuerza relativa del gel; el carácter viscoso está relacionado con el peso molecular y la fuerza de gelificación está definida por la relación entre $M / G$. El alginato empleado presenta una baja vicosidad (100-300 mPas), pero mayor contenido y longitud de bloques G (57\%), que de $M(17 \%)$, lo que aumenta la fuerza del gel, evitando deformaciones o hidrólisis, por factores externos. Un estudio que corrobora la acción benéfica del uso de polímeros sobre inoculantes biológicos fue el realizado por Pereira et al. (2010); en esta investigación, se registró que el polímero, no identificado, del inoculante comercial Stoller ${ }^{\circledR}$ protege la viabilidad de B. japonicum CPAC 15 y BR29 y conserva la promoción de crecimiento vegetal ejercida por estas cepas, para el cultivo de soya, en presencia de los fungicidas Tegran ${ }^{\circledR}$ y Derosal ${ }^{\circledR}$.

Estos resultados demuestran que el alginato actúa como un agente adherente y protectante que mitiga la acción inhibitoria de los fungicidas Carbendazim ${ }^{\circledR}$ y Fludioxonil ${ }^{\circledR}$, hasta las 24 horas de secado; por tal razón, el uso de polímeros representa una herramienta biotecnológica de gran importancia para disminuir los efectos perjudiciales de agroquímicos sobre grupos microbianos de potencial agroindustrial. 

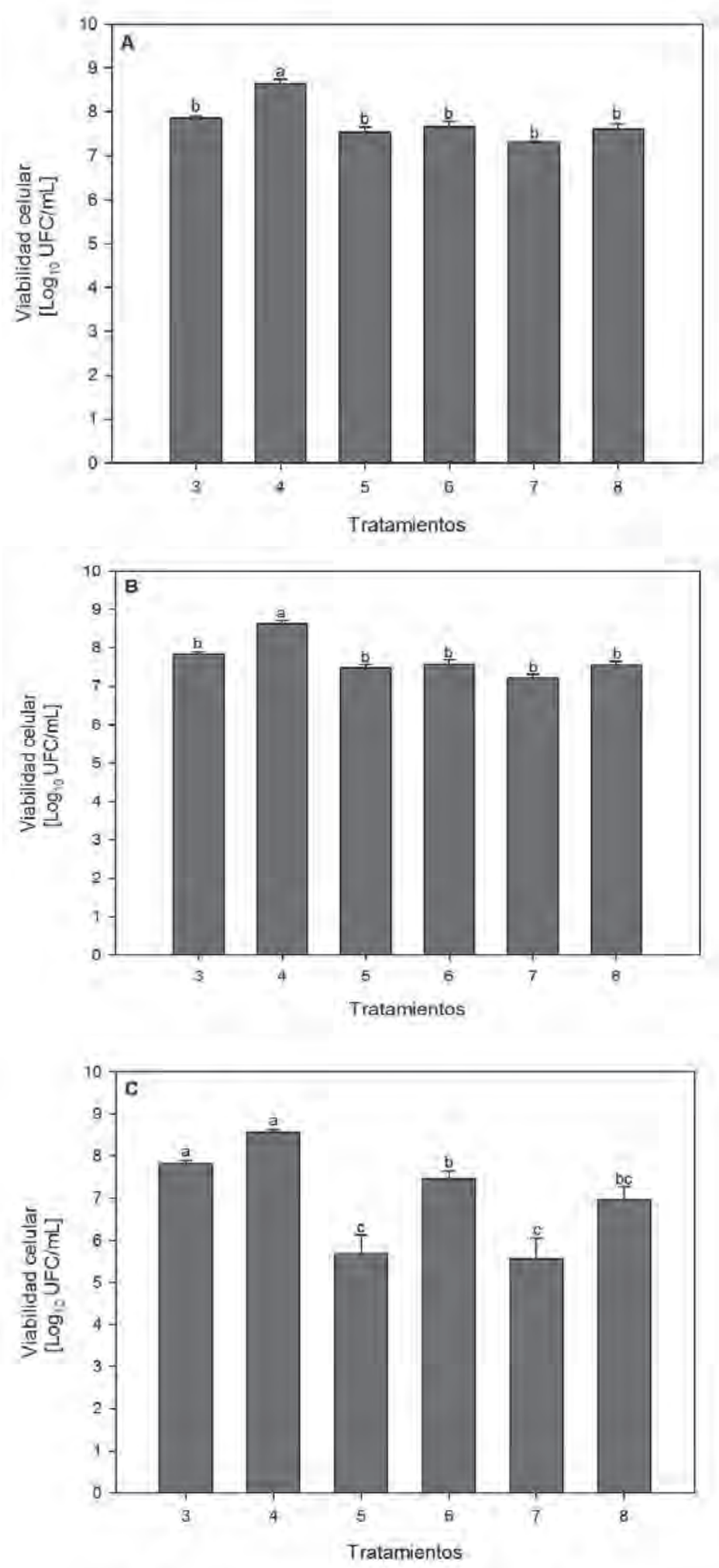

Figura 1. Efecto del polímero sobre la interacción entre fungicida y $B$. japonicum, a través de diferentes tiempos de secado. 1A) hora 0 ; 1B) hora 24; 1C) hora 48. En los controles químicos (T1 y T2) no se evidenció crecimiento microbiano. La barra de errores representa \pm la desviación estándar. Las letras indican grupos sub-homogéneos obtenido usando el test HSDTukey. 
Conflictos de intereses: El manuscrito fue preparado y revisado con la participación de todos los autores, quienes declaramos que no existe ningún conflicto de intereses que ponga en riesgo la validez de los resultados presentados. Financiación: Este estudio fue financiado por el Ministerio de Agricultura y Desarrollo dentro de la red de cultivos transitorios y agroindustriales de la Corporación Colombiana de Investigación Agropecuaria.

\section{BIBLIOGRAFÍA}

1. AHEMAD, M.; KHAN, M.S. 2012a. Alleviation of fungicide-induced phytotoxicity in greengram [Vigna radiata (L.) Wilczek] using fungicide-tolerant and plant growth promoting Pseudomonas strain. Saudi J. Biol. Sci. (Saudi Arabia). 19(4):451-459.

2. AHEMAD, M.; KHAN, M.S. 2012b. Effect of pesticides on plant growth promoting traits of greengramsymbiont, Bradyrhizobium sp. strain MRM6. Bull. Environ. Contam. Toxicol. (United States). 86(4):384388.

3. BASHAN, Y.; BASHAN, L.E.; PRABHU, S.R.; HERNANDEZ, J.P. 2013. Advances in plant growth-promoting bacterial inoculant technology: formulations and practical perspectives (19982013). Plant and Soil. (Netherlands). 378(1-2):1-33.

4. CASSÁN, F.; PERRIGA, D.; SGROYA, V.; MASCIARELLIA, O.; PENNAB, C.; LUNA, V. 2009. Azospirillum brasilense Az39 and Bradyrhizobium japonicum E109, inoculated singly or in combination, promote seed germination and early seedling growth in corn (Zea mays L.) and soybean (Glycine max L.). Eur. J. Soil Biol. (Italy). 45:28-35.

5. CORTÉS-PATIÑO, S.; BONILLA, R. 2015. Polymers selection for a liquid inoculant of Azospirillum brasilense based on the Arrhenius thermodynamic model. Afr. J. Biotechn. (Kenya). 14(33):2547-2553.

6. COVARRUBIAS, S.A.; DE-BASHAN, L.E.; MORENO, M.; BASHAN, Y. 2012. Alginate beads provide a beneficial physical barrier against native microorganisms in wastewater treated with immobilized bacteria and microalgae. Appl. Microbiol. Biotechnol. (Germany). 93:2669-2680.

7. DAYAMANI, K.J.; BRAHMAPRAKASH, G.P. 2014. Influence of form and concentration of the osmolytes in liquid inoculants formulations of plant growth promoting bacteria. Int. J. Scient. Res. Publ. (Malaysa). 4(7):1-6.
8. DESHWAL, V.K.; SINGH, S.B.; KUMAR, P.; CHUBEY A. 2013. Rhizobia unique plant growth promoting Rhizobacteria: A Review. Int. J. Life Scienc. (Nepal). 2(2):74-86.

9. DOYLE, M.; BEUCHAT, L.; MONTVILLE, T. 2001. Microbiología de los alimentos fundamentos y fronteras. 1 ed. Ed. Acribia (España). 816p.

10. GIONGO, A.; BENEDUZI, A.; GANO, K.; VARGAS, L.K.; UTZ, L.; PASSAGLIA, L.M.P. 2013. Characterization of plant growth-promoting bacteria inhabiting Vriesea gigantea Gaud. and Tillandsia aeranthos (Loiseleur) L.B. Smith (Bromeliaceae). Biota Neotrop. (Brazil). 13(3):80-85.

11. GOMBOTZ, W.R.; WEE, S.F. 2012. Protein release from alginate matrices. Advanc. Drug Delivery Rev. (Netherlands). 64:194-205.

12. IMFELDA, G.; VUILLEUMIERB, S. 2012. Measuring the effects of pesticides on bacterial communities in soil: A critical review. Eur. J. Soil Biol. 49:22-30.

13. JIANYA, S.U.; YE-CHENG, W.; SHU-KUN, Z.; XIU-BEI, R. 2014. Antifungal agents against Aspergillus niger for rearing rice leaffolder larvae (Lepidoptera: Pyralidae) on artificial diet. J. Econ. Entomol. (United States). 107(3):1092-1100.

14. JOHN, R.P.; TYAGI, R.D.; BRAR, S.K.; SURAMPALLI, R.Y.; PREVOST, D. 2011. Bio-encapsulation of microbial cells for targeted agricultural delivery. Crit. Rev. Biotechnol. (United Kingdom). 31:211-226.

15. KEUM, Y.S.; LEE, H.R.; KIM, J.H. 2010. Effects of pesticides on the bacterial production of pyrrolnitrin. J. Agric. Food Chem. (Nigeria). 58(9):5531-5537.

16. LEE, K.Y.; MOONEY, D.J. 2012. Alginate: Properties and biomedical applications. Progress in Polymer Science. (United Kingdom). 37(1):106-126.

17. LO, C.C. 2010. Effect of pesticides on soil microbial community. J. Environ. Sci. Health B. (United Kingdom). 45:348-359.

18. MIÑAMBRES, G.G.; CONLES, M.Y.; LUCINI, E.I.; VERDENELLI, R.A.; MERILES, J.M.; ZYGADLO, J.A. 2010. Application of thymol and iprodione to control garlic white rot (Sclerotium cepivorum) and its effect on soil microbial communities. World J. Microbiol. Biotechnol. (Netherlands). 26(1):161-170. 
19. MISHRA, G.; KUMAR, N.; GIRI, K.; PANDEY, S. 2013. In vitro interaction between fungicides and beneficial plant growth promoting rhizobacteria. Afr. J. Agr. Res. (Nigeria). 8(45):5630-5633.

20. NGUYEN, T.H.N.; BRECHENMACHER, L.; ALDRICH, J.T.; CLAUSS, T.R.; GRITSENKO, M.A.; HIXSON, K.K.; LIBAULT, M.; TANAKA, K.; YANG, F.; YAO, Q.; PAŠA-TOLIĆ, L.; XU, D.; NGUYEN, H.T.; STACEY, G. 2012. Quantitative phosphoproteomic analysis of soybean root hairs inoculated with Bradyrhizobium japonicum. Mol. Cell Proteomics. (United States). 11:1140-1155.

21. PEREIRA, C.E.; MOREIRA, F.M. de S.; OLIVEIRA, J.A.; CALDEIRA, C.M. 2010. Compatibility among fungicide treatments on soybean seeds through film coating and inoculation with Bradyrhizobium strains. Agronomy (United States). 32(4):585-589.

22. PII, Y.; MIMMO, T.; TOMASI, N.; TERZANO, R.; CESCO, S.; CRECCHIO, C. 2015. Microbial interactions in the rhizosphere: beneficial influences of plant growthpromoting rhizobacteria on nutrient acquisition process. A review. Biol. Fertil. Soils (Germany). 51(4):403-415.

23. RIVERA, D.; OBANDO, M.; BARBOSA, H.; ROJASTAPIAS, D.; BONILLA, R. 2014. Evaluation of polymers for the liquid rhizobial formulation and their influence in the Rhizobium-Cowpea interaction. Universitas Scientiarum. (Colombia). 19(3):265-275.

24. ROJAS-TAPIAS, D.; VERA, M.; RIVERA, D.; KLOEPPER, J.; BONILLA, R. 2013. Evaluation of three methods for preservation of Azotobacter chroococcum and Azotobacter vinelandii. Universitas Scientiarum. (Colombia). 18(2):129-139.

25. ROJAS-TAPIAS, D.; ORTEGA, O.; RIVERA, D.; BONILLA, R. 2015. Preservation of Azotobacter chroococcum vegetative cells in dry polymers. Universitas Scientiarum. (Colombia). 20(2):201-207.
26. ROJAS-TAPIAS, D.; MORENO-GÁLVAN, A.; PARDODÍAZ, S.; OBANDO, M.; RIVERA, D.; BONILLA, R. 2012. Effect of inoculation with plant growthpromoting bacteria (PGPB) on amelioration of saline stress in maize (Zea mays). Applied Soil Ecology. (Netherlands). 61:264-272.

27. ROMERO-PERDOMO, F.A.; MORENO-GALVÁN, A.; CAMELO-RUSINQUE, M.; BONILLA, R. 2015. Efecto de la carragenina sobre Azotobacter chroococcum en semillas de algodón peletizadas con un fungicida. Rev. Agron. Noroeste Argent. (Argentina). 35(1):2932.

28. RUBENS, J.C.; SILVA, R.; HUNGRIA, M. 2009. Nitrogen fixation with the soybean crop in Brazil: Compatibility between seed treatment with fungicides and bradyrhizobial inoculants. Symbiosis. (Netherlands). 48(1-3):154-163.

29. VALENCIA, R.A.; LIGARRETO, G. 2010. Análisis de la interacción soya-cepa (Bradyrhizobium japonicum) $\mathrm{x}$ ambiente, en oxisoles de la orinoquia colombiana. Agron. Col. (Colombia). 28(3):361-371.

30. VELOUKAS, T.; KALOGEROPOULOU, K.; MARKOGLOU, A.N.; KARAOGLANIDIS, G.S. 2014. Fitness and competitive ability of Botrytis cinerea field isolates with dual resistance to SDHI and Qol fungicides, associated with several $s d h B$ and the cytb G143A mutations. Phytopathology. (United States). 104(4):347-356.

31. VINCENT, L.M. 1970. A manual for the practical study of root-nodule bacteria. IBP Handbook no. 15. Ed. Blackwell Scientific (United Kingdom). 164p.

32. ZHANG, Y.; LAMM, R.; PILLONEL, C.; LAM, S.; XU, J.R. 2002. Osmoregulation and fungicide resistance: the Neurospora crassa os-2 gene encodes a HOG1 mitogen-activated protein kinase homologue. Appl. Environ. Microbiol. (United States). 68:532-538.

Recibido: Febrero 10 de 2015

Aceptado: Septiembre 16 de 2015

Cómo citar:

Romero-Perdomo, F.A.; Camelo, M.; Bonilla, R. 2015. Respuesta de Bradyrhizobium japonicum a la adición de alginato en presencia de fungicidas peletizados en semillas de soya. Rev. U.D.C.A Act. \& Div. Cient. 18(2): 359-364. 